\title{
Study of the bone behavior around a neck preserving short stem implant: bone densitometric analysis over a span of two years
}

\author{
Tarek Abdel Shafy ${ }^{1}$, Adel Sayed ${ }^{2}$, and Ahmed H. Abdelazeem ${ }^{1}{ }^{*}$ \\ 1 Kasralainy Hospital, Faculty of Medicine, Cairo University, Cairo, Egypt \\ 2 Arab Contractors Hospital, Arab Contractors Medical Center, Cairo, Egypt
}

Received 25 June 2016, Accepted 23 August 2016, Published online 7 October 2016

\begin{abstract}
Purpose: Study the bone mineral density (BMD) changes and the remodelling process after implantation of a neck preserving short stem implant over a period of two years.

Methods: Using specific patients' selection criterion, a prospective study was done including 26 patients. All were operated upon by a single surgeon using the MiniHipTM, (Corin, Cirencester, UK). Mean age was 42.5 years. Clinical and radiological evaluation was done. Periprosthetic bone density was measured by DEXA. First scan was obtained within 10 days after surgery and served as a baseline for comparison.

Results: The mean pre-operative Harris Hip score of 37.8 increased to 95.1 points two years post-operatively. BMD in the overall periprosthetic area showed a significant reduction during the first three months. Restoration to the original levels was reached in all zones except the most proximal zones at one year. A net increase was detected $(+3 \%)$ after two years.

Conclusion: The neck preserving MiniHip short stem implant has proven to be a bone-friendly design. Significant bone remodeling process continues after the first year. Although bone resorption in the greater trochanteric region is still a problem, however, it has proven that the BMD in all the other periprosthetic regions including the calcar and the lesser trochanteric regions, are subjected to bone formation process over a period of two years.
\end{abstract}

Key words: MiniHip, Short stem, DEXA, Periprosthetic remodelling, Neck preserving.

\section{Introduction}

The demographics of patients with hip disorders have changed over time. Patients are younger with high activity demands and have a longer life expectancy [1]. Periprosthetic bone resorption of the proximal femur is one of the main problems that affects the implants' survival especially in young generations. To avoid this, implants that mimic the physiological load transfer are required. Load transfer depends on the implant geometry, size, and degree of stiffness, type and extent of coating, as well as implant stability and osseointegration to the host bone [2].

Recently, many different designs of short femoral stems have been introduced. These designs are focused on more proximal load transfer and a sound physiological behavior as well as reduction of the diaphyseal fixation [3]. Albanese et al. suggested that shortening the femoral stem might promote complete proximal load transmission and, consequently, greater physiological stress distribution [4]. A biomechanical study by Whiteside et al., in 1995, confirmed the advantage of preserving the neck of femur during hip replacement because the forces encountered during weight-bearing are transferred more homogenously to the proximal femur when the femoral neck is preserved. Furthermore, the study revealed the importance of the femoral neck in increasing the rotation stability of the implant [5].

According to this information, we started using one of the "partial collum designs" or "neck preserving" short stem implants [3] in our institution since 2009; MiniHipTM (Corin, Cirencester, UK) on a specific type of patient. We conducted this study, on a specific group of patients, aiming at studying the bone behavior or response to these implants through examining the changes in the bone mineral densities (BMD) in the first two years after implantation. Hypotheses were: (1) bone resorption occurs in the first three months, afterwards; the process of bone formation starts and reaches its maximum

\footnotetext{
*Corresponding author: ahmed.abdelazeem@kasralaainy.edu.eg 
at one year, afterwards only minor changes will occur; (2) neck preservation will reduce the neck resorption process in the calcar and the lesser trochanteric regions.

\section{Patients and methods}

Between August 2009 and May 2015, a prospective study was conducted on 26 patients who had a unilateral total hip replacement using a single short stem hip implant (ceramic on ceramic MiniHipTM, Corin, Cirencester, UK). The study was conducted to examine the bone behavior around this short stem. All patients were followed up clinically and radiologically for two years. The study was approved by the Institutional Review Board (IRB) of the Department of Trauma and Orthopaedics, Arab Contractors Hospital, Cairo.

The study was conducted on patients with special demographics excluding all patients with radiologically detectable osteopenia, abnormal deviation of the neck (anteversion $>25^{\circ}$, coxa valga $>150^{\circ}$ or coxa vara $<115^{\circ}$ ), pathological fracture, previous metaphyseal osteotomies or deformities, and contralateral hip disease or replacement (Table 1). All surgeries were done using epidural anesthesia in a lateral position using the posterolateral approach and by the same surgeon (TS) in a single hospital.

\section{Clinical evaluation}

Harris hip score (HHS) [6] was used for pre- and postoperative clinical evaluation for all patients at three months, six months, one year, and two years of follow-up.

\section{Radiological evaluation}

Pre-operative radiographs were analyzed for femoral bone quality based on the Dorr classification [7]. The angle of the neck resection level was verified through templating; the mid-neck resection level was used. Femoral stem alignment was assessed on the immediate anteroposterior (AP) radiographs of the pelvis and classified as neutral, valgus, or varus. A neutral alignment was considered when the distal polished part of the stem was in contact with and parallel to the endosteum of the lateral cortex, and the upper medial part of the stem was in contact with the calcar area. A valgus alignment was considered when the stem was resting its distal polished part on the medial cortex. A varus alignment was considered when the stem was anchored by the tip to the lateral cortex, and its upper medial part was not in contact with the medial calcar area. To assess stability, length measurements from the superior tip of the greater trochanter to the distal tip of the implant were compared between immediate post-operative and long-term follow-up visits.

\section{Duel Energy X-ray Absorptiometry (DEXA)}

The periprosthetic bone density was measured by DEXA scan using specialized orthopedic software (Lunar Prodigy Primo version $P R+351125)$. The scanning procedure, as well
Table 1. Patient's demographics.

\begin{tabular}{llc}
\hline Number & & 26 \\
\hline Age & Range & $21-50$ \\
& Mean & 42.5 \\
Sex & Males & 20 \\
& Females & 6 \\
Side & Right & 15 \\
& Left & 11 \\
Causes of & Degenerative hip diseases & 22 \\
replacement & Fracture neck femur - Garden IV & 3 \\
& Revision of loose resurfacing & 1 \\
& component & $19-37$ \\
BMI $\left(\mathrm{kg} / \mathrm{m}^{2}\right)$ & Range & 27.3 \\
& Mean \\
\hline
\end{tabular}

as the positioning of the patient and the degree of leg rotation, were standardized for all patients to guarantee high measurement accuracy. The DEXA scan was obtained within seven to 10 days after surgery and served as a baseline for the subsequent scans [8]. The proximal femur was divided into seven zones (Regions of Interest (ROIs)) according to Gruen et al [9]. The bone mineral content $(\mathrm{g})$, area $\left(\mathrm{cm}^{2}\right)$, and bone mineral density (BMD) $\left(\mathrm{g} / \mathrm{cm}^{2}\right)$ were calculated in the seven ROIs (Figure 1). The BMD was measured laterally (Zones 1, 2, and 3) and medially (Zones 5, 6, and 7) around the stem and at least $1 \mathrm{~cm}$ distal to the tip of the stem (Zone 4). Zones 1-7 were combined to determine the total periprosthetic BMD.

Further scans were obtained at three months, six months, 12 months, and 24 months post-operatively. The postoperative change in BMD was assessed by comparing the subsequent $\mathrm{BMD}$ value with the $\mathrm{BMD}$ value measured immediately post-operatively. The difference was expressed as the percent change versus the baseline value.

\section{Statistical analysis}

The statistical analysis was performed by a qualified biostatistician. Data were analyzed using the Statistical Program for Social Science (SPSS) version 18.0. Quantitative data were expressed as means \pm standard deviation (SD). Qualitative data were expressed as frequencies and percentages. A probability ( $p$-value) $<0.05$ was considered significant, a $p$-value $<0.001$ was considered highly significant, and a $p$-value $>0.05$ was considered non-significant.

\section{Results}

\section{Clinical results}

The mean pre-operative HHS of 37.8 points $(\mathrm{SD} \pm 14.9)$ increased to 95.1 points $(\mathrm{SD} \pm 3.9$ ) two years post-operatively (Table 2). In all cases, thigh pain was not observed at the six month follow-up evaluation. Highly significant differences between the pre-operative and the six month, one year, and two year HHSs using a paired sample $t$-test, which results in $p$-values $<0.001$, were observed. 

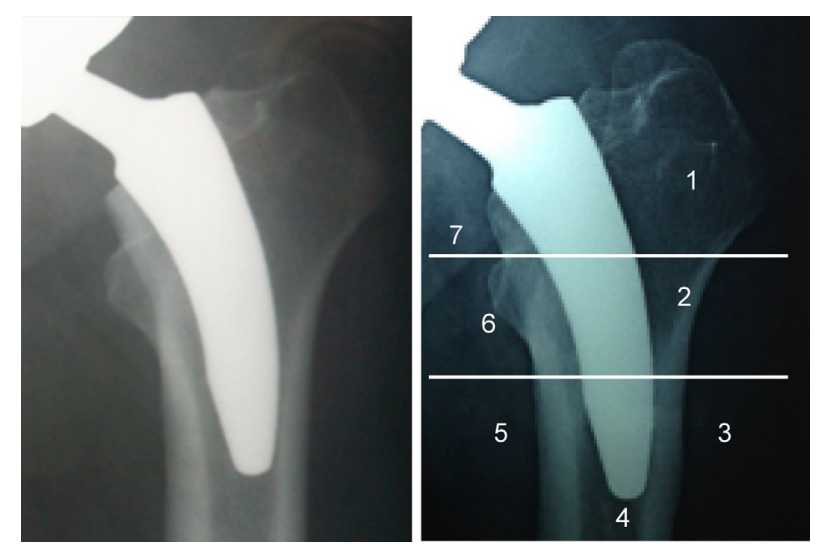

Figure 1. Regions of interest (RIOs) according to Gruen's zones.

Two complications were reported, which we think did not affect the results of our study. Acute Deep Venous Thrombosis (DVT) occurred in one male patient three weeks post-operation and a dislocation occurred in another case. That was a 48-yearold female patient who experienced a traumatic posterior dislocation after falling on the ground six months postoperatively. The dislocation was managed by closed reduction under general anesthesia and bed rest for six weeks.

\section{Radiological results}

The immediate post-operative and subsequent radiographic findings are summarized in Table 3.

\section{Densitometry results}

The mean results of the BMD in the seven ROIs were compared between immediate post-operative baseline values and subsequent follow-up results (Table 4 and Figure 2).

In ROI 1, highly significant bone loss $(-14 \%)$ was observed in the first three months after surgery $(p<0.001)$. Despite a partial restoration of BMD after two years, the final value $(-3 \%)$ was less than the initial value, which still represented a significant change $(p<0.05)$.

In ROI 2, an initial, highly significant reduction in the BMD value occurred $(-9 \%)$, followed by a progressive increase in the BMD to the level of the baseline value, which was not significant in the first year $(2 \%)$, but was significant after two years $(5 \%)$, compared to the baseline value $(p<0.05)$.

In ROI 3, the BMD initially decreased $(-2 \%)$ in the first three months; however, this loss was regained six months after surgery. The final BMD after two years $(7 \%)$ resulted in a highly significant difference from the baseline value.

In ROI 4, an initial loss of BMD occurred, but it was regained after two years. A slight, gradual increase was noted between 12 months and 24 months. The final BMD was less than $1 \%$ greater than the baseline value.

In ROI 5, a highly significant initial loss of BMD occurred $(-5 \%)$, followed by a constant increase in BMD until it was regained one year post-operatively, and at two years, the BMD gain was statistically significant $(4 \%)$.
Table 2. Differences between the pre- and post-operative Harris hip scores (HHSs).

\begin{tabular}{lcrcc}
\hline \multicolumn{1}{c}{ HHS } & Mean & \pm SD & Mean Diff. & $p$-value \\
\hline Pre & 37.8 & 14.9 & & \\
After 6 months & 83.1 & 12.4 & -45.4 & $<0.001$ \\
After 1 year & 92.7 & 3.3 & -53.9 & $<0.001$ \\
After 2 years & 95.1 & 3.7 & -59.1 & $<0.001$ \\
\hline
\end{tabular}

Table 3. Summary of immediate and post-operative follow-up radiographic results.

\begin{tabular}{|c|c|}
\hline Radiographic parameter & Findings \\
\hline \multicolumn{2}{|l|}{ Femoral morphology } \\
\hline Dorr classification: no. (\%) & Total no. $=26$ hips $(100 \%)$ \\
\hline Type A & $10(38 \%)$ \\
\hline Type B & $16(62 \%)$ \\
\hline Type $C$ & 0 \\
\hline $\begin{array}{l}\text { Femoral component } \\
\text { alignment: } \text { no. }(\%)\end{array}$ & Total no. $=26$ hips $(100 \%)$ \\
\hline Neutral & $25(96 \%)$ \\
\hline Varus & $1(4 \%)$ \\
\hline $\begin{array}{l}\text { Postop. limb length discrepancy } \\
\text { mean and range }(\mathrm{cm})\end{array}$ & $\begin{array}{l}\text { Mean }=0.8 \mathrm{~cm} \\
\text { Range }=(0.5-1 \mathrm{~cm})\end{array}$ \\
\hline Calcar atrophy: no. (\%) & No. $=26$ hips at 2 years postop. \\
\hline Round-off & $18(70 \%)$ \\
\hline Loss of calcar height & $1(4 \%)$ \\
\hline Loss of calcar thickness & $1(4 \%)$ \\
\hline Heterotrophic ossification: & No. $=26$ hips at 2 year postop. \\
\hline No. $(\%)$ & $2(8 \%)$ \\
\hline Grade I & $1(4 \%)$ \\
\hline Grade II & 0 \\
\hline Grade III & $1(4 \%)$ \\
\hline Grade IV & 0 \\
\hline $\begin{array}{l}\text { Stem osseointegration (stable): } \\
\text { no. (\%) }\end{array}$ & No. $=26$ hips at 2 years postop. \\
\hline $\begin{array}{l}\text { Bone bridging (spot welds) } \\
\text { in at least one Gruen zone }\end{array}$ & 23 hips $(88 \%)$ \\
\hline Cortical hypertrophy & 3 hips $(12 \%)$ \\
\hline Radiolucent lines: no. (\%) & 0 \\
\hline $\begin{array}{l}\text { Stem tip reactive sclerotic line: } \\
\text { no. (\%) }\end{array}$ & $1(4 \%)$ \\
\hline
\end{tabular}

In ROI 6, BMD loss occurred (-9\%) during the first three months $(p<0.001)$, but it was partially regained at 12 months. The BMD gradually increased from 12 to 24 months to reach $2 \%$ above the initial value, which does not represent a significant difference from the baseline value.

In ROI 7, BMD loss $(-13 \%)$ occurred during the first six months $(p<0.001)$; gradual restoration was observed, but it did not reach the initial value after two years $(-3 \%$, not significant).

The net mean BMD decreased significantly during the first three months $(-7 \% ; p<0.001)$ but returned to the initial value by one year. Thereafter, additional changes were observed. The final BMD after two years was almost 3\% higher than the immediate post-surgical value making a statistically significant difference (Figure 3). 


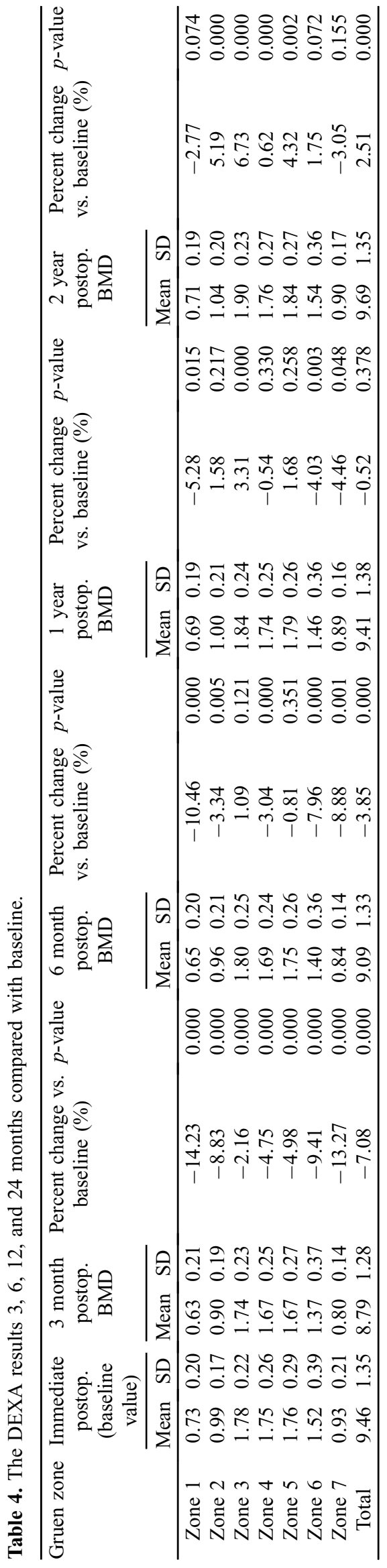

\section{Discussion}

Short stem hip implants have been classified by Van Oldenrijk et al. into three categories: column, partial column, and trochanter-sparing stem designs. From the clinical point of view, they found unsatisfactory survival rate and accordingly, did not support the use of the column designs. However, they reported promising medium-term results with the other two designs [3]. From the radiological point of view, few reports studied the bone remodeling process and changes in bone densitometry around the different short stem designs [10-13].

Concerning the partial column (neck preserving) designs, Lerch et al, in 2012, using the Metha short stem (B. Braun Aesculap, Tuttlingen, Germany) and the DEXA analysis, studied the changes in the bone densitometry after implantation in 25 patients. They found that BMD in the greater trochanteric region decreased significantly two years post-operatively. Minor changes were seen in the distal regions while in the lesser trochanteric region, significant increase was reported by $12.9 \%$ at two years follow-up. In the calcar region, BMD exceeded the baseline value by $6.1 \%$. They concluded that the DEXA analysis showed load concentration on the medial part of the proximal femur and considered this an important guarantee for successful longterm results [10]. Similarly, at one year follow-up, Jahnke et al, in 2014, using the same implant in 40 patients, found a decreased bone density in ROIs 1, 4, and 7 and increased density in ROIs 2, 3, 5, and 6. They reported a hypertrophic bone remodeling process primarily in the lesser trochanter region by $6 \%$ [11].

A more proximal stress shielding was reported when studying the trochanter-sparing stem designs. In a retrospective BMD analysis following implantation of the Mayo (Zimmer, Winterthur, Switzerland) short stem, a significant decrease in ROIs 1, 6, and 7 was observed (ranging 14.4-17.9\%), while much less affection was observed in ROIs 2-5 [12]. They concluded that there had been distal bony ingrowth with proximal resorption. Recently, Freitag et al when comparing the Fitmore short stem with CLS cementless straight stem (both Zimmer, Winterthur, Switzerland) noted also a stress protection of the proximal femur following trochanter-sparing short stem implantation. However, this stress protection was much less when compared with conventional stem type [13].

Using specific selection criterion, this prospective study was carried out to examine the bone behavior following the implantation of partial column design short stem (MiniHipTM, Corin, Cirencester, UK). The MiniHip stem depends on metaphyseal 'fit without fill' for maximum bone conservation. Its medial curve follows the curve of the medial calcar for physiological load transfer. It is made of a titanium alloy with a hydroxyapatite coating on a titanium plasma spray in the proximal area of the stem.

DEXA is believed to be an efficient method for the evaluation of bone remodeling after hip arthroplasty. Several cross-sectional $[14,15]$ and longitudinal $[16,17]$ DEXA studies have been conducted after cemented and uncemented prothesis. In the present study, we used a longitudinal DEXA 


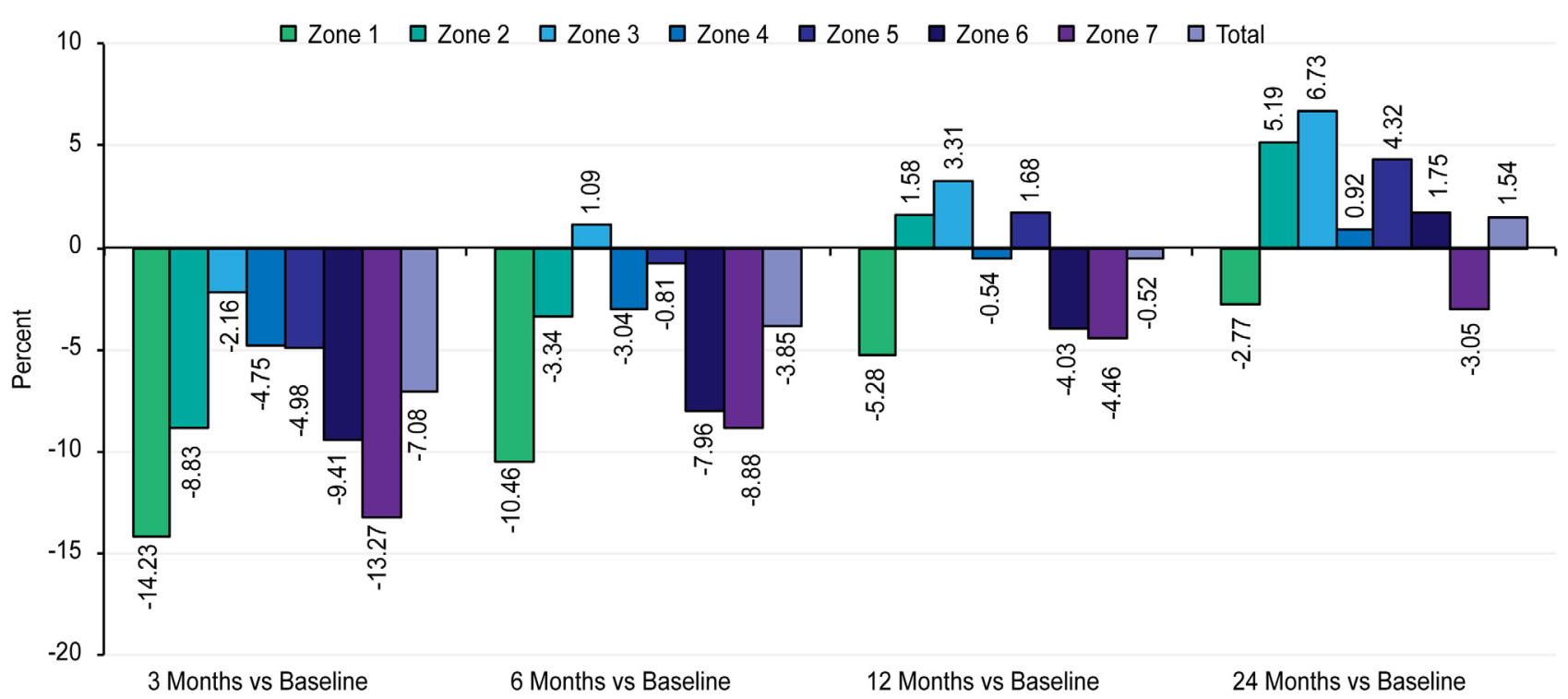

Figure 2. Bar chart showing the DEXA results 3, 6, 12, and 24 months as compared with baseline.

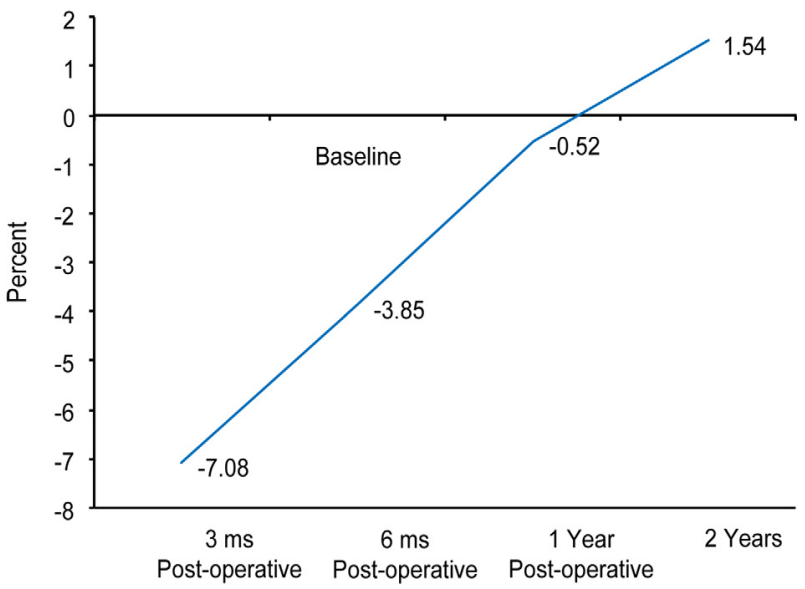

Figure 3. Graph showing the net changes in the BMD over a span of 2 years.

study protocol to assess bone remodeling. The initial BMD value obtained one week post-operatively was recorded as the baseline value to estimate the changes in the periprosthetic BMD in subsequent follow-up scans.

After surgery, the BMD in the overall periprosthetic area showed a significant reduction during the first three months. The most astounding reduction was recorded in the most proximal zones (ROIs 1 and 7), while the smallest reduction was observed in Zone 3, where the lateral part of the implant pushes against the lateral cortex. However, at 12 months, all the zones have regained some of their lost bone densities. In Zones 2, 3, 4, and 5 the BMD had reached a plateau near the initial values recorded shortly after the index procedure, with the highest increment recorded in Zone 3 laterally $($ mean $+3.3 \%)$. Statistically significant negative values were still recorded in the most proximal Zones 1, 6, and 7 with an average bone loss of $4-5 \%$ of the baseline values. When the two year BMD value after surgery was compared with the baseline value, two zones exhibited significantly lower BMDs (Zones 1 and 7), two zones had significantly higher BMDs (Zones 2 and 6), the change in BMD was highly significant in one zone (Zone 3), and two zones had non-significant increases in BMD (Zones 4 and 5). The net mean periprosthetic BMD was not statistically significant at one year but turned to be have a significant increase $(+3 \%)$ at two years follow-up.

Our results were consistent with the previous records in the literature, denoting a process of bone resorption immediately after the surgery in all the periprosthetic areas. This was reversed, after the first three months post-operatively, to a process of bone formation that continues till two years. Significant changes occurred after the first year, especially in the lesser trochanteric region (Zone 6), which turned from a negative to a positive value after the first year. These changes did not support our hypothesis, present in the current literature [10-13], that only minor changes occur after the first year.

Bone resorption detected in Zone 7 (the lower neck) as well as the rounding off phenomena detected in $70 \%$ of the cases using the plain radiographs (Figure 4) over a span of two years follow-up, indicated stress shielding of this area. Contrary to this, increased BMD in the lesser trochanteric area indicated load concentration in the calcar area. This finding supports our hypothesis that neck preservation is an important factor for bone preservation in the calcar and the lesser trochanteric regions.

A remarkable observation in the current study is that when osseointegration between the bone and prosthesis is achieved, restoration of the periprosthetic bone is achieved.

Unification of the surgical procedure, using the DEXA, and careful patient's selection are considered the main strengths of our study. However, the short follow-up period as well as the limited number of patients are considered the main weakness points.

In conclusion, the neck preserving MiniHip short stem implant has proven to be a bone-friendly design. Although bone resorption in the greater trochanteric region is still a 
(A)

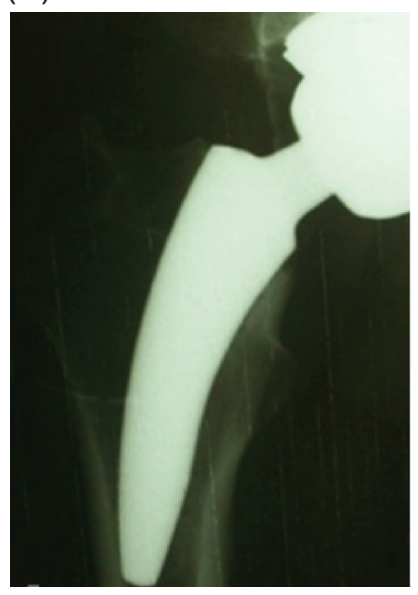

(B)

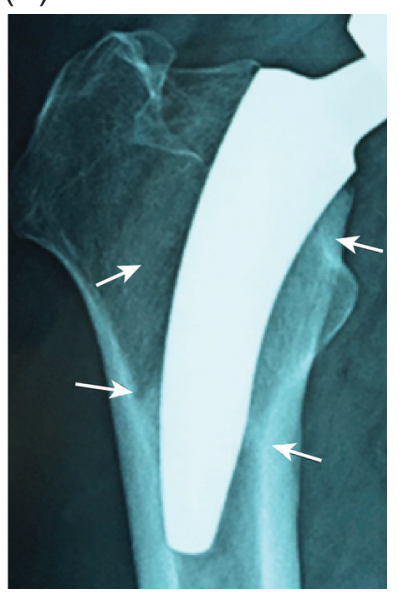

Figure 4. Anteroposterior radiographs of 40-year-old male: (A) immediate post-operative and (B) two years follow-up. The stem is considered in neutral alignment. Two years later; osseointegration evidenced by increased trabecular density bridging from lateral and medial cortex to the stem (spot welds - lower arrows) as well as rounding of the lower neck (upper arrows).

problem, however, it has proven that the BMD in all the other periprosthetic regions including the calcar and the lesser trochanteric regions, are subjected to bone formation process over a period of two years.

\section{Conflict of interest}

The authors declare no conflict of interest in relation with this paper.

\section{References}

1. Chidambaram R, Cobb AG (2009) Change in the age distribution of patients undergoing primary hip and knee replacements over 13 years - an increase in the number of younger men having hip surgery. J Bone Joint Surg Br, 91(SUPP I), $152-152$.

2. Walker P, Roberston D (1988) Design and fabrication of cementless hip stems. Clin. Orthop. Relat. Res., 235, 25-34.

3. Falez F, Casella F, Papalia M. (2015). Current concepts, classification, and results in short stem hip arthroplasty. Orthopedics, 38(3), S6-S13.

4. Albanese CV, Rendine M, De Palma F, Impagliazzo A, Falez F, Postacchini F, Santori FS (2005) Bone remodelling in THA: a comparative DXA scan study between conventional implants and a new stemless femoral component. A preliminary report. HIP, 16, 9-15.

5. Whiteside LA, White SE, McCarthy DS (1995) Effect of neck resection on torsional stability of cementless total hip replacement. Am J Orthop (Belle Mead, NJ), 24(10), 766-770.

6. Harris WH (1969) Traumatic arthritis of the hip after dislocation and acetabular fractures: treatment by mold arthroplasty. J Bone Joint Surg Am, 51(4), 737-755.

7. Dorr LD, Faugere MC, Mackel AM, Gruen TA, Bognar B, Malluche HH (1993) Structural and cellular assessment of bone quality of proximal femur. Bone, 14(3), 231-242.

8. Kröger H, Miettinen H, Arnala I, Koski E, Rushton N, Suomalainen O (1996) Evaluation of periprosthetic bone using dual-energy X-ray absorptiometry: precision of the method and effect of operation on bone mineral density. J. Bone Miner. Res., 11(10), 1526-1530.

9. Gruen T, Mcneice G, Amstutz H (1979) "Modes of failure" of cemented stem-type femoral components: a radiographic analysis of loosening. Clin Orthop Relat Res, 141, 17-27.

10. Lerch M, von der Haar-Tran A, Windhagen H, Behrens BA, Wefstaedt P, Stukenborg-Colsman CM (2012) Bone remodelling around the Metha short stem in total hip arthroplasty: a prospective dual-energy X-ray absorptiometry study. Int Orthop, 36(3), 533-538.

11. Jahnke A, Engl S, Altmeyer C, Jakubowitz E, Seeger JB, Rickert M, Ishaque BA (2014) Changes of periprosthetic bone density after a cementless short hip stem: a clinical and radiological analysis. Int Orthop, 38(10), 2045-2050.

12. Chen HH, Morrey BF, An KN, Luo ZP (2009) Bone remodeling characteristics of a short-stemmed total hip replacement. J Arthroplasty, 24(6), 945-950.

13. Freitag T, Hein MA, Wernerus D, Reichel H, Bieger R (2016) Bone remodelling after femoral short stem implantation in total hip arthroplasty: 1-year results from a randomized DEXA study. Arch Orthop Trauma Surg, 136(1), 125-130.

14. Trevisan C, Bigoni M, Cherubini R, Steiger P, Randelli G, Ortolani S (1993) Dual X-ray absorptiometry for the evaluation of bone density from the proximal femur after total hip arthroplasty: analysis protocols and reproducibility. Calcif Tissue Int, 53(3), 158-161.

15. Niinimaki T, Jalovaara P (1995) Bone loss from the proximal femur after arthroplasty with an isoelastic femoral stem: BMD measurements in 25 patients after 9 years. Acta Orthop Scand, 66(4), 347-351.

16. Marchetti ME, Steinberg GG, Greene JM, Jenis LG, Baran DT (1996) A prospective study of proximal femur bone mass following cemented and uncemented hip arthroplasty. J Bone Miner Res, 11(7), 1033-1039.

17. Nakamura K (1996) Measurement of periprosthetic bone mineral density after cementless hip arthroplasty by dual energy X-ray absorptiometry: longitudinal and cross-sectional evaluation. J Orthop Sci, 1(2), 113-122. 